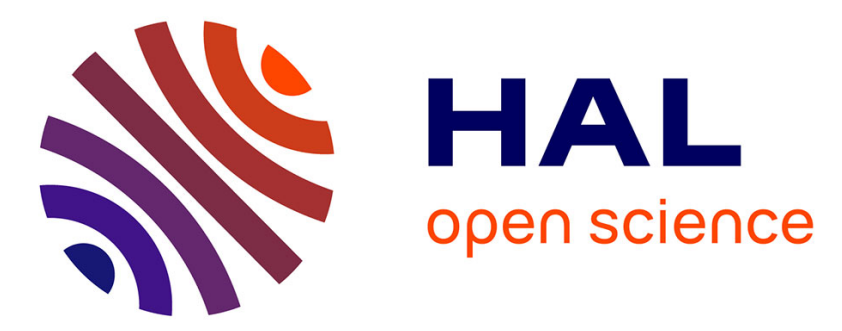

\title{
LES MOBILITÉS SOUS CONTRAINTES DES MĖRES SEULES AVEC ENFANT(S) : ANALYSE DANS LE CADRE DE LA BRETAGNE (FRANCE)
}

Frédéric Leray, Raymonde Séchet

\section{- To cite this version:}

Frédéric Leray, Raymonde Séchet. LES MOBILITÉS SOUS CONTRAINTES DES MÈRES SEULES AVEC ENFANT(S) : ANALYSE DANS LE CADRE DE LA BRETAGNE (FRANCE). Philippe Gerber, Samuel Carpentier. Mobilités et modes de vie: vers une recomposition de l'habiter, Presses universitaires de Rennes, pp.69-88, 2013, 978-2-7535-2249-7. halshs-01345576

\section{HAL Id: halshs-01345576 \\ https://shs.hal.science/halshs-01345576}

Submitted on 14 Jul 2016

HAL is a multi-disciplinary open access archive for the deposit and dissemination of scientific research documents, whether they are published or not. The documents may come from teaching and research institutions in France or abroad, or from public or private research centers.
L'archive ouverte pluridisciplinaire HAL, est destinée au dépôt et à la diffusion de documents scientifiques de niveau recherche, publiés ou non, émanant des établissements d'enseignement et de recherche français ou étrangers, des laboratoires publics ou privés. 


\section{LES MOBILITÉS SOUS CONTRAINTES DES MÈRES SEULES AVEC ENFANT(S) : ANALYSE DANS LE CADRE DE LA BRETAGNe (France)}

Frédéric LERAY et Raymonde SÉcheT

Représentant environ une famille française sur cinq en 2005, les familles monoparentales pourraient être considérées comme des familles ordinaires si elles n'étaient pas plus vulnérables que les autres familles avec enfants (Chardon, Daguet, Vivas, 2008). Très majoritairement dirigées par des femmes (c'est aujourd'hui le cas de $85 \%$ des familles monoparentales françaises) dont les salaires moyens sont inférieurs à ceux des hommes, les familles monoparentales sont nombreuses à disposer de revenus du travail ne permettant pas de dépasser le seuil de pauvreté. Cette pauvreté des familles monoparentales et l'impact des politiques sociales sont fréquemment analysés. Il n'en est pas de même des aspects relatifs aux conditions de logement, aux mobilités résidentielles et aux mobilités quotidiennes des mères seules qui sont l'objet d'une recherche menée en Bretagne ${ }^{1}$ et dont nous présentons ici les premiers résultats.

Cette recherche s'inscrit dans le prolongement d'études précédemment réalisées pour l'Observatoire national de la pauvreté (Séchet, David, Quintin, 2002) et pour la Caisse Nationale d'Allocations Familiales (David et al., 2003; David et al., 2004). Ces études reposaient sur l'hypothèse que la fréquence de la pauvreté des familles monoparentales trouve son origine dans un espace de vulnérabilité combinant des dimensions économiques, sociétales, territoriales abordées dans la première partie de l'article. La deuxième partie du texte porte sur les conditions de logement et les mobilités résidentielles des femmes seules avec enfant(s). L'ampleur des changements de logement au moment du passage à la monoparentalité permet d'affirmer que la monoparentalité entraîne fréquemment des parcours résidentiels régressifs qui, à leur tour, peuvent être constitutifs de la vulnérabilité des familles monoparentales féminines. Les mobilités quotidiennes sont ensuite analysées pour elles-mêmes et dans leurs articulations avec les mobilités résidentielles. Bien que les situations individuelles soient diverses,

1. Cette recherche est financée par la Région Bretagne (ARED nº 06007534). 
ces mobilités quotidiennes sont globalement largement contraintes par le fait de devoir faire face en solo ${ }^{2}$.

\section{DES MÈRES SEULES DANS UN ESPACE DE VULNÉRABILITÉ}

Après le rappel de quelques éléments de contexte démographique, cette première partie présente l'idée d'espace de vulnérabilité des familles monoparentales puis la méthodologie de la recherche en cours.

\section{Des familles monoparentales plus nombreuses et toujours vulnérables}

La proportion des familles monoparentales dans le total des familles avec enfants de moins de 25 ans n'a cessé de croître en France (fig. 1) comme en Bretagne (fig. 2). La définition usuelle de la famille monoparentale est celle de l'Institut national de la statistique et des études économiques : « La famille monoparentale est une famille composée d'un père ou d'une mère vivant seule avec son ou ses enfants. » L'Insee retient un critère de co-résidence. Le parent isolé ne partage pas sa résidence principale avec un conjoint ou avec une autre personne, qu'elle ait ou non un lien de parenté.

Ces familles monoparentales étant surtout de petite taille (Ramonet, 2009), la part des enfants vivant avec un seul de leurs parents est inférieure à celle des familles monoparentales dans le total des familles avec enfants. Elle a toutefois plus que doublé depuis les années 1970. Dans le même temps, les modalités de constitution des familles monoparentales ont évolué. Pendant longtemps, le veuvage découlant de décès précoces, tant des pères que des mères, était la première cause de monoparentalité, suivie par les naissances d'enfants chez les « filles-mères ». La hausse de l'espérance de vie ainsi que la diffusion de la contraception et la légalisation de l'avortement (loi Neuwirth en 1967, loi Veil en 1975) ont fortement réduit la part des célibataires et veuves dans le total des mères seules. Comme dans le même temps on a assisté au déclin de la signification du mariage (Bumpass, 1994), ce sont maintenant surtout les ruptures familiales divorces et séparations - qui sont à l'origine des situations de monoparentalité. Celles-ci ne sont d'ailleurs souvent qu'une étape dans les parcours familiaux à laquelle une recomposition familiale vient mettre un terme.

Figure 1: Évolution du nombre de familles monoparentales dans le total des familles avec enfants en France (en \%)

2. Les expressions « faire face seule » ou « faire face en solo » sont utilisées pour désigner toutes les occasions et situations dans lesquelles les mères doivent assumer l'ensemble des tâches quotidiennes du ménage, qu'elles bénéficient ou non de l'aide financière et/ou organisationnelle de l'ancien conjoint. 


\begin{tabular}{|c|c|c|c|c|c|c|}
\hline & 1968 & 1975 & 1982 & 1990 & 1999 & 2005 \\
\hline $\begin{array}{c}\text { Nombre de familles } \\
\text { monoparentales }\end{array}$ & 719700 & 776260 & 887040 & 1175444 & 1493700 & 1760000 \\
\hline $\begin{array}{c}\text { \% de familles } \\
\text { monoparentales }\end{array}$ & 9,3 & 9,4 & 10,2 & 13,2 & 17,4 & 19,8 \\
\hline $\begin{array}{c}\text { \% des enfants de } \\
\text { moins de 25 ans } \\
\text { vivant dans une famille } \\
\text { monoparentale }\end{array}$ & 7,7 & 8,1 & 8,9 & 11,2 & 15 & 17,5 \\
\hline
\end{tabular}

Champ: ensemble des familles avec enfant(s) de moins de 25 ans et enfants de moins de 25 ans vivant dans une famille monoparentale.

Source: Insee, recensements de la population, 2005.

Figure 2: Évolution du nombre de familles bretonnes selon le nombre d'enfants de moins de 25 ans

\begin{tabular}{|c|c|c|c|}
\hline $\begin{array}{c}\text { Nombre d'enfants } \\
\text { de moins de 25 ans }\end{array}$ & Type de ménage & $\mathbf{1 9 9 9}$ & $\mathbf{2 0 0 5}$ \\
\hline \multirow{2}{*}{1} & Monoparental & 29743 & 36428 \\
& Couple & 119323 & 124089 \\
& Part des familles monoparentales (en \%) & 20 & 23 \\
\hline \multirow{2}{*}{2} & Monoparental & 17049 & 21361 \\
& Couple & 144321 & 148918 \\
& Part des familles monoparentales (en \%) & 11 & 13 \\
\hline \multirow{3}{*}{3} & Monoparental & 5791 & 6709 \\
& Couple & 63899 & 60077 \\
& Part des familles monoparentales (en \%) & 8 & 10 \\
\hline \multirow{2}{*}{4 et plus } & Monoparental & 1643 & 1756 \\
& Couple & 14308 & 13151 \\
& Part des familles monoparentales (en \%) & 10 & 12 \\
\hline \multirow{2}{*}{ Ensemble des ménages } & Monoparental & 53956 & 66254 \\
& Couple & 341851 & 346235 \\
& Part des familles monoparentales (en \%) & 14 & 16 \\
\hline
\end{tabular}

Source: Mickaël Ramonet, Insee-Octant, $n^{0}$ 115, janvier 2009.

Le passage de la monoparentalité « du registre de la déviance à celui de la variance des formes familiales » (Lefaucheur, 1985, p. 210) n'a pas empêché la vulnérabilité des familles monoparentales. En 2003, $27 \%{ }^{3}$ d'entre elles vivent en dessous du seuil de pauvreté contre $12 \%$ des couples avec enfant(s) (Blanpain, 2007). Parce qu'elles sont dirigées par des femmes et parce qu'elles ne comportent qu'un parent, les familles monoparentales avec enfants sont prises

3. Champ: Les personnes vivant dans un ménage avec enfant(s) qui ne sont pas étudiantes et dont la personne de référence à moins de 60 ans. Le taux de pauvreté monétaire prend en compte le niveau de vie après prestations familiales, aides au logement et minima sociaux. Les ménages pauvres sont ceux qui disposent de ressources inférieures à $60 \%$ du revenu médian. 
dans un ensemble de contraintes spécifiques. Dès lors, la plus grande fréquence de la pauvreté qui les frappe s'inscrirait dans un "espace de vulnérabilité » (Séchet et al., 2002, p. 259), hypothèse qui emprunte, en l'adaptant, la réinterprétation que Watts et Bohle (1993) ont fait de l'espace des capacités (capabilities) des individus - ce que les personnes sont en mesure de faire - théorisé par A. Sen (Paulo, 2006). La vulnérabilité des mères seules, quelle que soit la singularité des situations personnelles, émergerait à la rencontre entre:

- dimension familiale, avec notamment des contraintes d'emploi du temps inhérentes à l'obligation de devoir faire face seule aux activités et tâches du quotidien, pour soi et pour ses enfants. Ces contraintes peuvent d'autant plus rendre difficile la pérennisation de réseaux sociaux que le modèle familial biparental reste dominant;

- dimension économique, puisque les mères seules doivent pourvoir aux besoins de la famille alors que le marché du travail est moins favorable aux femmes (salaires plus faibles, emplois sous-qualifiés, contrats à durée déterminée et emplois à temps partiel plus fréquents);

- dimension territoriale, qui comporte deux niveaux. Le premier, relatif aux ressources du territoire dans lequel s'inscrivent les espaces de vie personnels, notamment en matière d'emploi et de logement, est un facteur de différenciation spatiale entre les familles monoparentales : c'est à ce niveau que se dessinent les disparités dans les taux de pauvreté économique des familles monoparentales (David, Quintin, Séchet, 2002). Le second niveau est celui des espaces de vie des femmes seules avec enfants, celui des pratiques spatiales et des déplacements entre domicile, lieu de travail, courses, etc. (Séchet et al., 2002, p. 259) (fig. 3).

La combinaison de ces trois dimensions est potentiellement source de pauvreté économique (articulation entre les dimensions familiales et économiques), de marginalisation (articulation entre dimension économique et dimension spatiale), d'exclusion sociale (articulation entre dimension familiale et dimension territoriale). Précarité sociale, pauvreté économique, pauvreté des conditions de vie interagissent dans l'espace de vulnérabilité, au risque d'une déstabilisation cumulative (Naves, 2001, p. 14).

Figure 3: L'" espace de vulnérabilité » des mères isolées 


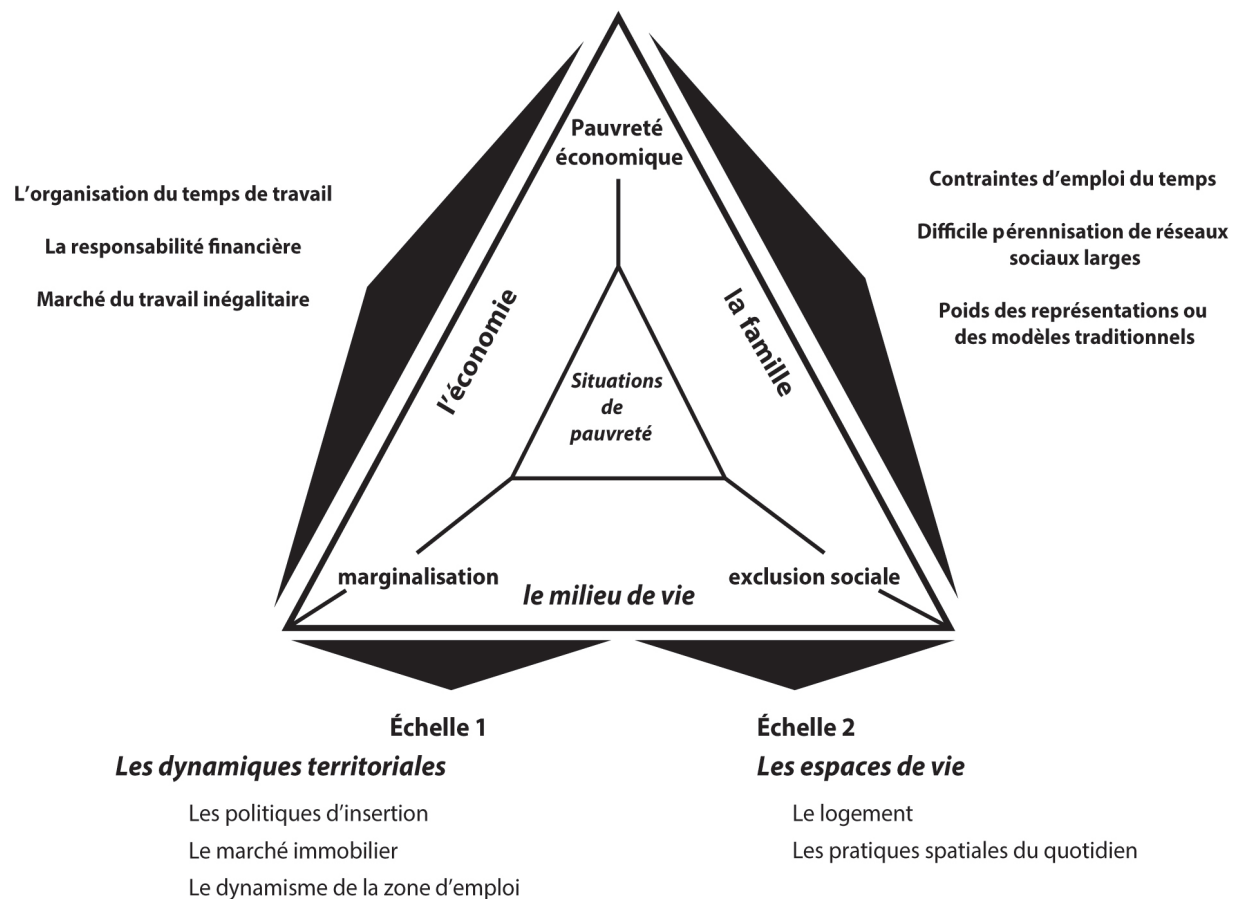

Source : Séchet R., David O., Quintin, P. 2002. Les familles monoparentales et la pauvreté. In Les Travaux de l'Observatoire national de la pauvreté et de l'exclusion 2001-2002, Chapitre 3, Paris : La Documentation française, 247-290 d'après M.Watts et H. Bohle. 1993. The space of vulnerability: the causal structure of hunger and famine. Progress in Human Geography, vol. 17.11 : 43-67.

\section{L'étude des mobilités résidentielles et quotidiennes des mères seules en Bretagne}

Les mobilités des mères seules avec enfants peuvent être appréhendées dans le cadre problématique de l'existence d'un espace de vulnérabilité selon une double hypothèse: d'une part, l'entrée en monoparentalité et les contraintes inhérentes à l'obligation de faire face seule peuvent générer des changements résidentiels importants et, d'autre part, l'organisation des déplacements quotidiens est fonction de contraintes temporelles, économiques, sociétales, les spatialités induites pouvant, en retour, générer un surcroît de vulnérabilité. Pour mieux connaître ces mobilités et leur articulation, une enquête par questionnaire a été réalisée avec le soutien de la Fédération bretonne des Caisses d'Allocations Familiales. Les cinq CAF bretonnes ${ }^{4}$ se sont d'abord chargées de l'envoi

4. Une caisse en Ille-et-Vilaine, Côtes d'Armor, Morbihan, deux dans le Finistère. 
de 4000 lettres d'informations demandant aux mères seules avec enfants de donner leur accord pour participer à l'enquête, comme cela a été exigé par la Commission nationale de l'informatique et des libertés (CNIL). Les communes d'envoi ont été sélectionnées en fonction de la répartition des familles monoparentales entre villes-centres, communes monopolarisées, multipolarisées et rurales $^{5}$ (Leray, Séchet, 2009): en Bretagne, $70 \%$ des familles monoparentales résident dans un pôle urbain, $13 \%$ dans une commune multipolarisée, $6 \%$ dans une commune monopolarisée, $11 \%$ dans une commune rurale. L'hypothèse de l'inégale capacité des espaces de vie à offrir plus ou moins de ressources (caractéristiques du marché de l'emploi, réseaux de transports, offre de services de garde...) a justifié le choix de mener les observations dans tous les types d'espaces. Le nombre de questionnaires envoyés dans chaque commune a donc varié précisément en fonction de ces pourcentages. Les CAF ont ensuite assuré l'envoi des questionnaires aux 1495 femmes ayant donné leur accord. À la date du $1^{\text {er }}$ septembre 2008, 902 questionnaires avaient été retournés, soit un taux de réponse de $60 \%$. Au total, après saisie de l'ensemble des réponses, 800 questionnaires valables ${ }^{6}$ ont été soumis au traitement informatique des données.

Les répondantes présentent des caractéristiques proches de celles observées aux niveaux national et régional ${ }^{7}$, tant pour les modes de formation de la famille monoparentale (11 \% par naissance d'un enfant alors que la mère ne vivait pas en couple avant, $78 \%$ par désunion, qu'il s'agisse de la rupture d'union libre $(46 \%)$ ou d'un divorce (32\%), et enfin $10 \%$ par décès du conjoint) que pour l'âge. Par ailleurs, la durée moyenne des situations de monoparentalité observées est de 6,3 années.

Une phase qualitative s'est faite sous la forme d'entretiens semi-directifs avec comme objectif une meilleure compréhension de la dimension spatiale du vécu de la monoparentalité. Pour que cela soit possible, la Fédération bretonne des Caisses d'Allocations Familiales a autorisé la présence sur le questionnaire d'un encart permettant de contacter les mères qui ont donné leur accord. Suite à l'analyse de 800 questionnaires, 26 femmes seules avec enfant(s) ont été interviewées. Quatre thématiques principales ont été abordées: le changement de logement, les déplacements au quotidien, la gestion du temps et la situation monoparentale « vécue».

\footnotetext{
5. Les définitions sont celles de l'INSEE: une "ville-centre » est une commune qui abrite plus de $50 \%$ de la population de l'unité urbaine; une « commune monopolarisée » est une commune appartenant à la couronne périurbaine d'une aire urbaine; une "commune multipolarisée " se situe hors des aires urbaines (pôle urbain et couronne périurbaine) et au moins $40 \%$ de sa population ayant un emploi travaille dans plusieurs aires urbaines; une " commune rurale » est une commune n'appartenant pas à une unité urbaine.

6. Un questionnaire mal rempli doit être écarté plutôt que de saisir des informations incertaines.

7. Les données comparatives sont issues du recensement de 2006.
} 


\section{DES SITUATIONS ET PARCOURS RÉSIDENTIELS FRAGILISÉS}

Les caractéristiques de statut et de localisation du logement des mères seules avec enfants sont le reflet de la fragilisation qui intervient au moment de la constitution des familles monoparentales et dont découle souvent une régression des parcours résidentiels.

\section{Une forte présence des mères seules avec enfants dans le parc social}

Dans une région où l'accession à la propriété est fortement valorisée et où les deux tiers des ménages sont propriétaires de leur logement (Lévy, 2008), les mères isolées ne le sont que dans 34,8 \% des cas. Celles-ci sont à l'inverse beaucoup plus souvent locataires, soit dans le parc locatif privé pour plus du quart d'entre elles, soit, surtout, dans le parc locatif social : c'est le cas de 36,4 \% des enquêtées contre seulement 10,3\% des ménages bretons en 2006 (fig. 4). Les mères les plus représentées dans le logement social sont les mères inactives (une sur deux) et les jeunes (58 \% des moins de 25 ans contre $30 \%$ des mères de 45 ans et plus résident dans un logement social). À l'inverse, les mères les plus avancées dans le cycle de vie sont plus souvent propriétaires (la moitié des mères seules de 45 ans et plus).

Figure 4: Répartition des mères isolées et des ménages bretons selon les statuts d'occupation (en \%)

\begin{tabular}{|c|c|c|}
\hline Statut d'occupation & Mères isolées* & Ménages bretons** \\
\hline Propriétaire & 34,8 & 66 \\
\hline Locataire privé & 27,1 & 21,9 \\
\hline Locataire H.L.M & 36,4 & 10,3 \\
\hline Hébergement & 1,6 & 1,8 \\
\hline Total & $\mathbf{1 0 0}$ & $\mathbf{1 0 0}$ \\
\hline
\end{tabular}

* Source: enquête " les mères isolées en Bretagne ", Université Rennes 2/Fédération bretonne des Caisses d'Allocations Familiales.

* Source: Insee, Recensement 2006, exploitations principales.

Ces spécificités des statuts résidentiels découlent de choix largement contraints. Dans les deux-tiers des cas, les mères isolées ont déménagé au moment du changement familial qui a débouché sur la monoparentalité. Et à défaut de pouvoir alors élaborer une réelle stratégie résidentielle, les mères isolées s'adaptent. Aux motifs économiques, qui peuvent concerner n'importe quel ménage, s'ajoutent ici des contraintes de nature familiale. C'est le cas lorsque les mères choisissent de résider près du domicile de leur ex-conjoint afin de pérenniser les relations des enfants avec leur père: "Pour que ma fille puisse voir plus souvent son papa, je me suis rapprochée de son domicile. J'ai alors 
rencontré de grosses difficultés pour trouver un logement décent » (Isabelle, séparée, 45 ans, un enfant).

\section{Les ruptures familiales: un moment charnière dans les parcours résidentiels des mères seules avec enfants}

$\mathrm{Au}$ moment du changement familial, s'instaure un temps d'arbitrage entre contraintes économiques, exigences familiales, conciliation des temps... Les raisons qui motivent le déménagement - l'obligation financière, le besoin de résider dans un logement indépendant et la volonté de changer de cadre de vie (une mère isolée sur cinq pour chacun de ces motifs de déménagement) mais aussi l'exigence de l'ex-conjoint ou la volonté de se rapprocher de la famille proche (une mère seule sur huit pour l'un et l'autre cas) - mettent en évidence la vulnérabilité des mères seules au moment où leur situation familiale change. La mobilité résidentielle touche aussi bien les salariées que les non salariées, les cadres que les ouvrières (entre $60 \%$ et les deux-tiers des mères dans tous les cas). La monoparentalité génère donc des changements résidentiels importants quel que soit le niveau de vie des mères isolées. Toutefois, les possibilités de choix du logement varient selon les situations personnelles.

Un test de khi-deux ${ }^{8}$ montre que les relations de dépendance à la mobilité résidentielle sont très significatives pour l'origine de la monoparentalité, le lieu de vie et le statut d'occupation antérieurs, et qu'elles sont significatives pour l'âge des mères alors qu'elles ne le sont pas ou peu pour l'âge des enfants, le type de garde parentale ou la PCS des mères. Les plus susceptibles de changer de logement sont celles qui ont connu une désunion (près des trois-quarts des mères divorcées et séparées ont changé de logement) ainsi que les plus jeunes (alors que les trois quarts des mères âgées de moins de 25 ans au moment de l'entrée en monoparentalité ont changé de logement, cela n'a concerné que $56 \%$ des mères de 45 ans et plus). À l'inverse, les veuves, plus souvent propriétaires et élevant des enfants plus âgés que les autres, sont moins concernées par un changement de logement: «Je suis veuve mais quand cela m'est arrivé, j'avais déjà un emploi et j'étais propriétaire, et ma fille avait 14 ans. Je n'ai donc eu à trouver ni un emploi, ni un logement, ni un mode de garde pour mes enfants. C'est sûr que ma vie a changé mais je n'ai pas eu à affronter toutes ces difficultés. » (Élodie veuve, 50 ans, un enfant.) En ce qui concerne le statut du logement, résider dans un logement social incite moins souvent à la mobilité résidentielle (54 \% des mères résidant en logement social quittent leur logement au moment

8. Le test du khi-deux détermine si les réponses à deux questions fermées sont « statistiquement significatives » (indépendantes) selon le calcul suivant: Chi calculé= somme (Effectif Théorique - Effectif Réel)2 / Effectif Théorique. La relation est ensuite validée ou non selon la valeur de référence de chaque table du Chi-deux. 
du changement familial). Peut-être parce que, dans ce cas, les pères sont plus enclins à laisser le logement à l'ex-épouse ou compagne et que, surtout, celui-ci ne coûte pas trop cher. Et lorsque les mères isolées quittent un logement social, près des trois quarts d'entre elles vont dans un autre logement social. Pour les propriétaires, le passage à la monoparentalité a des conséquences variables : si les mères de catégories relativement aisées peuvent garder leur logement, éventuellement en rachetant la part de leur ex-conjoint, celles dont la situation financière et familiale ne le permet pas ou dont l'ex-conjoint l'exige doivent se résoudre à la vente du logement.

\section{La fréquence des parcours résidentiels régressifs}

Les parcours résidentiels des femmes qui ont déménagé au moment du changement familial sont souvent marqués par une régression en termes de type de logement et de statut de celui-ci ainsi que par un changement de commune de résidence.

Alors qu'antérieurement à leur situation de monoparentalité, près de $60 \%$ des mères isolées résidaient dans une maison individuelle, elles sont moins d'une sur quatre après le changement familial et cette part ne remonte que légèrement pour atteindre $30 \%$ des situations observées. À l'inverse, la part des mères isolées logeant dans un appartement croît fortement, passant de $40 \%$ à $76 \%$ au moment du changement familial pour ensuite diminuer légèrement $(70 \%$ des situations observées). En outre, au moment de la rupture qui a conduit à la monoparentalité, quelques femmes ont signalé avoir eu recours à des solutions précaires (mobil home, camping car). La maison individuelle faisant figure de norme résidentielle pour les familles avec enfants (CREDOC, 2004), les passages de la maison vers l'appartement peuvent être vécus négativement par les mères, et cela d'autant plus que ce changement de nature du logement est fréquemment associé à un passage du statut de propriétaire à celui de locataire. Mireille (divorcée, 41 ans) exprime cette idée:

« Passer d'une maison en campagne à l'appartement ici, c'est plus petit, ça manque d'un parc, il a fallu du temps pour s'y adapter, on n'a pas juste changé de logement, on change de vie tout court! »

La part des propriétaires diminue fortement (de $41 \%$ à $8 \%$ ) après l'entrée en monoparentalité avant de remonter ensuite pour atteindre près du quart des mères enquêtées, (fig. 5). À l'inverse, à l'autre extrémité des statuts résidentiels, la part de celles qui vivaient dans un logement social progresse fortement pour dépasser $40 \%$, cette part évoluant peu entre le moment de l'entrée en monoparentalité et la situation observée dans l'enquête, comme si les mères seules contraintes de rejoindre ce type de logement en devenaient captives. La part 
des mères hébergées double (de $5 \%$ à plus de $10 \%$ ) au moment du changement familial. Neuf fois sur dix, ces mères retournent chez leur(s) parent(s), soit que leur situation financière l'impose, soit que le trouble affectif engendré par le changement familial, surtout si celui-ci est soudain, induise le besoin de retrouver un cadre de vie familier et de bénéficier du soutien parental. Ces situations d'hébergement ne sont souvent que temporaires puisque moins de $2 \%$ des mères enquêtées sont hébergées au moment de l'enquête contre 10,8 \% après l'entrée en situation monoparentale. Au final, les passages du statut de propriétaire à celui de locataire, dans le parc privé comme dans le parc social, et du locatif privé vers le locatif social ainsi que la stabilité dans le parc social de la grande majorité de celles qui y étaient déjà sont des figures autrement plus fréquentes que le maintien dans le statut de propriétaire (fig. 6).

Figure 5: L'évolution des statuts résidentiels des mères seules avec enfants (en \%)

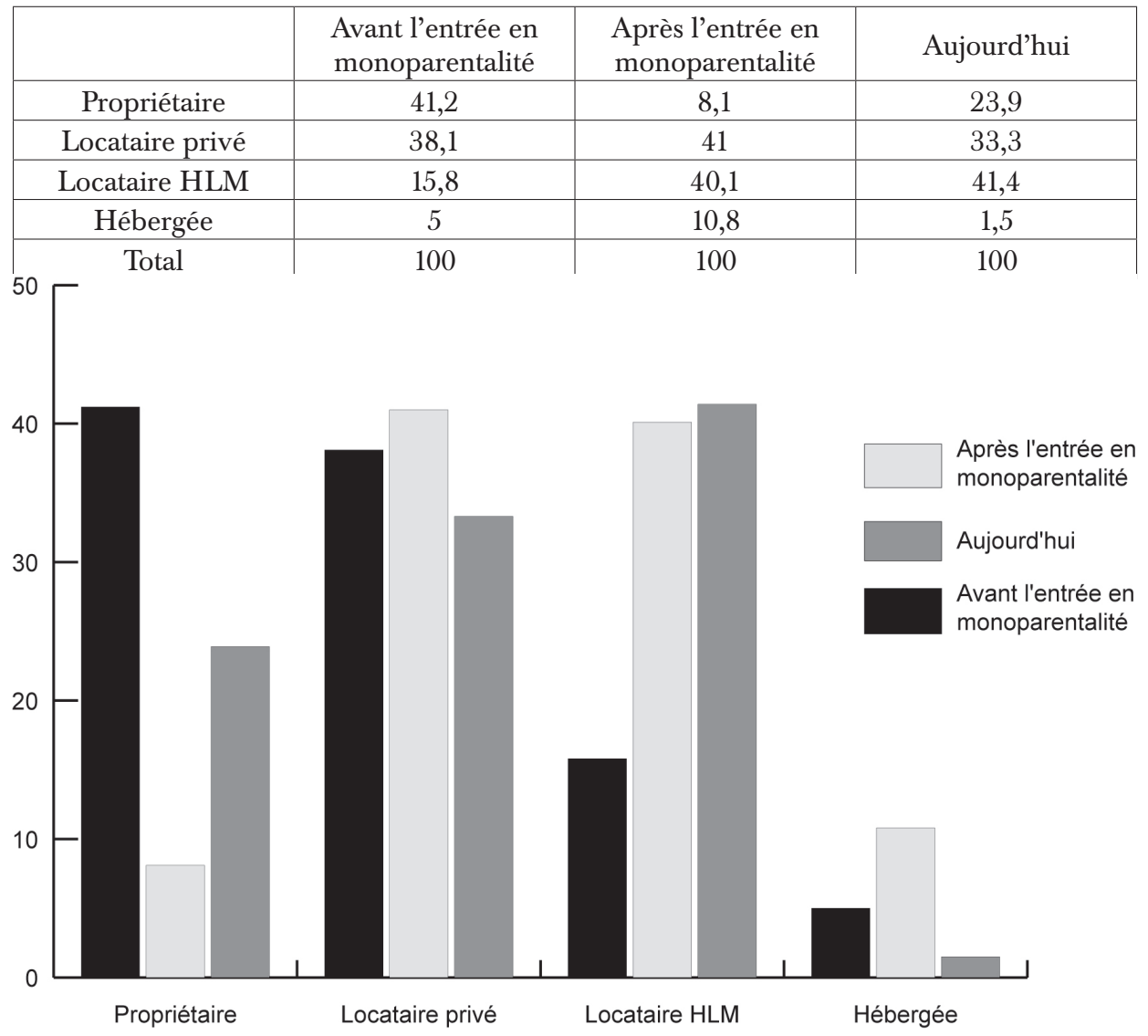

Note de lecture : « aujourd'hui » : au moment de l'enquête. 
Figure 6: Les comportements résidentiels selon les statuts d'origine (en \%)

\begin{tabular}{|c|c|c|c|}
\hline $\begin{array}{c}\text { Statut } \\
\text { d'occupation }\end{array}$ & $\begin{array}{l}\text { Avant l'entrée en } \\
\text { monoparentalité }\end{array}$ & $\begin{array}{l}\text { Après l'entrée en } \\
\text { monoparentalité }\end{array}$ & $\begin{array}{l}\text { Au moment de } \\
\text { l'enquête }\end{array}$ \\
\hline Propriétaire & $41,2 \%$ & $\begin{array}{c}\text { 6,8 \% - Propriétaire } \\
\text { 20,4 \% - Locataire privé } \\
\text { 10,9 \% - Locataire H.L.M } \\
\text { 3,1 \% - Hébergement }\end{array}$ & $\begin{array}{c}\text { 14,7 \% - Propriétaire } \\
\text { 14,1\% - Locataire privé } \\
\text { 11,6 \% - Locataire H.L.M } \\
\text { 0,7\% - Hébergement }\end{array}$ \\
\hline $\begin{array}{c}\text { Locataire } \\
\text { privé }\end{array}$ & $38,1 \%$ & $\begin{array}{c}\text { 0,6 \% - Propriétaire } \\
\text { 15,8 \% - Locataire privé } \\
\text { 15,1 \% - Locataire H.L.M } \\
\text { 6,6 \% - Hébergement }\end{array}$ & $\begin{array}{c}\text { 5,6 \% - Propriétaire } \\
\text { 15,2\% - Locataire privé } \\
\text { 16,7 \% - Locataire H.L.M } \\
0,6 \% \text { - Hébergement }\end{array}$ \\
\hline $\begin{array}{l}\text { Locataire } \\
\text { H.L.M }\end{array}$ & $15,8 \%$ & $\begin{array}{c}\text { 1,0 \% - Propriétaire } \\
\text { 2,1 \% - Locataire privé } \\
\text { 11,5 \% - Locataire H.L.M } \\
\text { 1,2 \% - Hébergement }\end{array}$ & $\begin{array}{c}\text { 2,9 \% - Propriétaire } \\
\text { 1,9\% - Locataire privé } \\
\text { 10,9\% - Locataire H.L.M } \\
0,0 \% \text { - Hébergement }\end{array}$ \\
\hline Hébergement & $5 \%$ & $\begin{array}{c}\text { 0,0 \% - Propriétaire } \\
\text { 1,8 \% - Locataire privé } \\
3,2 \% \text { - Locataire H.L.M } \\
0,0 \% \text { - Hébergement }\end{array}$ & $\begin{array}{c}0,6 \% \text { - Propriétaire } \\
\text { 1,6 \% - Locataire privé } \\
2,8 \% \text { - Locataire H.L.M } \\
0,0 \% \text { - Hébergement }\end{array}$ \\
\hline Total & 100 & 100 & 100 \\
\hline
\end{tabular}

Champ: mères isolées ayant dû changer de logement au moment de l'entrée en monoparentalité.

Les changements de logement s'accompagnent d'évolutions dans les localisations ${ }^{9}$. La part des mères vivant dans l'espace rural diminue, passant de $21 \%$ avant l'entrée en monoparentalité à $12 \%$ après le changement familial puis $10 \%$ au moment de l'enquête. C'est que les communes rurales offrent peu de logements sociaux et qu'elles sont moins bien desservies en transports collectifs que les communes urbaines. Faire face en solo exige souvent une proximité entre l'ensemble des lieux nécessaires à la vie quotidienne qui est difficile à satisfaire en milieu rural: «En m'installant à Rennes, c'était beaucoup plus pratique, il y a les transports en commun, il y a tous ces aspects-là que j'avais vu, je me disais qu'il faut mieux choisir une grande ville que plutôt se tourner vers la campagne où c'est beaucoup plus difficile, surtout quand on est seul. » (Véronique, divorcée, 41 ans, deux enfants.) Par ailleurs, la part des mères vivant dans une commune du périurbain éloigné augmente légèrement, aussi bien au moment du changement de situation familiale qu'ensuite, notamment parce que l'offre de logements locatifs privés y a progressé (DRE, 2004). Enfin, la part des mères isolées vivant dans les pôles urbains, où se trouve l'essentiel du parc locatif, aussi bien social

9. En raison du faible nombre de répondantes résidant dans une commune rurale et une commune multipolarisée, le choix a été fait de regrouper ces deux types de communes qui n'appartiennent ni aux pôles urbains ni aux couronnes périurbaines et de distinguer: les «pôles urbains - villescentres et périurbain proche -, les communes monopolarisées - périurbain éloigné -, l'espace rural - communes rurales et communes multipolarisées. 
que privé, augmente. Cette augmentation est plus limitée pour les villes-centre, où les locataires captives du parc HLM changent peu de logement, que dans le périurbain proche: c'est là que les propriétaires ou locataires du parc privé cherchent un logement social au moment du déménagement (immeubles récents, cadre de vie satisfaisant, souci de limiter le sentiment de régression sociale).

\section{FAIRE FACE EN SOLO: LES MOBILITÉS QUOTIDIENNES COMME REFLET D'ARBITRAGES ENTRE TRAVAIL ET ENFANTS?}

Les parcours résidentiels des mères seules avec enfants, les choix plus ou moins contraints des deux-tiers d'entre elles qui ont dû changer de logement influencent fortement les mobilités quotidiennes. Même si l'analyse des mobilités quotidiennes en relations avec les mobilités résidentielles a été faite de manière globale pour l'ensemble des mères seules avec enfants, cinq profils de comportements résidentiels ont fait l'objet d'une attention particulière: propriétaire avant l'entrée en monoparentalité et au moment de l'enquête, passage de propriétaire à locataire dans le parc privé, passage de propriétaire à locataire HLM, passage de locataire privé à locataire HLM, locataire HLM avant l'entrée en monoparentalité et au moment de l'enquête.

Figure 7: Interactions entre comportements résidentiels et pratiques spatiales du quotidien

\begin{tabular}{|c|c|c|c|c|c|c|}
\hline & \multicolumn{5}{|c|}{$\begin{array}{l}\text { Évolution du statut d'occupation : de la situation } \\
\text { résidentielle d'origine à celle d'aujourd'hui }\end{array}$} & \multirow[b]{2}{*}{ 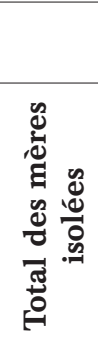 } \\
\hline & 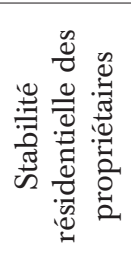 & 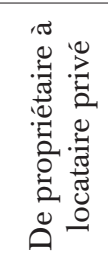 & 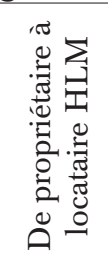 & 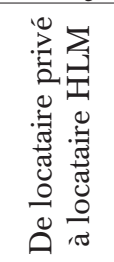 & 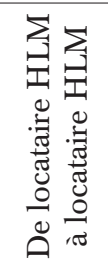 & \\
\hline \multicolumn{7}{|l|}{ Le cadre de vie } \\
\hline $\begin{array}{l}\text { Satisfaction du } \\
\text { logement }\end{array}$ & $60 \%$ & $27,4 \%$ & $22,4 \%$ & $30,2 \%$ & $25 \%$ & $38,6 \%$ \\
\hline $\begin{array}{l}\text { Satisfaction du } \\
\text { cadre de vie }\end{array}$ & $67,8 \%$ & $34,2 \%$ & $32,8 \%$ & $38,8 \%$ & $37,5 \%$ & $47,1 \%$ \\
\hline $\begin{array}{c}\text { «Sentiment » } \\
\text { de captivité au } \\
\text { logement }\end{array}$ & $12 \%$ & $27,4 \%$ & $40 \%$ & $37,9 \%$ & $33,9 \%$ & $27,1 \%$ \\
\hline \multicolumn{7}{|l|}{$\begin{array}{c}\text { La situation } \\
\text { professionnelle }\end{array}$} \\
\hline $\begin{array}{l}\text { Évolution de la vie } \\
\text { professionnelle }\end{array}$ & $63,6 \%$ & $57,5 \%$ & $71,7 \%$ & $63,2 \%$ & $71,4 \%$ & $65,4 \%$ \\
\hline
\end{tabular}




\begin{tabular}{|c|c|c|c|c|c|c|}
\hline $\begin{array}{c}\text { Changement } \\
\text { d'emploi } \\
\text { ou de formation }\end{array}$ & $10,3 \%$ & $11 \%$ & $23,3 \%$ & $28,7 \%$ & $25 \%$ & $19,6 \%$ \\
\hline $\begin{array}{l}\text { «Sentiment» } \\
\text { d'une restriction } \\
\text { professionnelle }\end{array}$ & $31,6 \%$ & $39,7 \%$ & $51,7 \%$ & $62,1 \%$ & $48,1 \%$ & $47,8 \%$ \\
\hline \multicolumn{7}{|l|}{$\begin{array}{l}\text { Les déplacements } \\
\text { au quotidien }\end{array}$} \\
\hline $\begin{array}{c}\text { Facilité de } \\
\text { déplacements }\end{array}$ & $43,6 \%$ & $38,4 \%$ & $43,1 \%$ & $31,3 \%$ & $43,1 \%$ & $41,5 \%$ \\
\hline $\begin{array}{c}\text { Distance entre } \\
\text { logement et le lieu } \\
\text { de travail }>12 \mathrm{~km}^{*}\end{array}$ & $54 \%$ & $41,5 \%$ & $51,5 \%$ & $34,6 \%$ & $38,1 \%$ & $49,1 \%$ \\
\hline $\begin{array}{c}\text { Utilisation } \\
\text { d'un véhicule } \\
\text { personnel pour le } \\
\text { lieu de travail }\end{array}$ & $99,1 \%$ & $93,2 \%$ & $95 \%$ & $76,7 \%$ & $73,2 \%$ & $81,6 \%$ \\
\hline $\begin{array}{c}\text { Sollicitation de } \\
\text { l'entourage pour } \\
\text { les déplacements } \\
\text { de la mère }\end{array}$ & $17,8 \%$ & $19,2 \%$ & $19,3 \%$ & $34,5 \%$ & $31,8 \%$ & $25,4 \%$ \\
\hline $\begin{array}{c}\text { Sollicitation } \\
\text { de l'entourage pour } \\
\text { les déplacements } \\
\text { de l'enfant }\end{array}$ & $44,1 \%$ & $43,8 \%$ & $33,1 \%$ & $33,7 \%$ & $36,4 \%$ & $46 \%$ \\
\hline \multicolumn{7}{|l|}{$\begin{array}{l}\text { Les modes } \\
\text { de garde** }\end{array}$} \\
\hline $\begin{array}{l}\text { Facilité pour trouver } \\
\text { un service de garde }\end{array}$ & $21,9 \%$ & $23,3 \%$ & $19 \%$ & $15,8 \%$ & $16,7 \%$ & $23,5 \%$ \\
\hline $\begin{array}{c}\text { Satisfaction de } \\
\text { l'offre de services } \\
\text { de garde }\end{array}$ & $28 \%$ & $10,9 \%$ & $16,7 \%$ & $14,7 \%$ & $9,3 \%$ & $23,1 \%$ \\
\hline \multicolumn{7}{|l|}{$\begin{array}{l}\text { Les réseaux } \\
\text { sociaux }\end{array}$} \\
\hline $\begin{array}{l}\text { Baisse des relations } \\
\text { amicales }\end{array}$ & $13,3 \%$ & $58,9 \%$ & $56,7 \%$ & $64 \%$ & $62,5 \%$ & $58,2 \%$ \\
\hline $\begin{array}{l}\text { Augmentation des } \\
\text { relations familiales }\end{array}$ & $66,4 \%$ & $67,1 \%$ & $56,7 \%$ & $68,2 \%$ & $66,7 \%$ & $68,6 \%$ \\
\hline $\begin{array}{c}\text { Sentiment } \\
\text { d'isolement }\end{array}$ & $56,4 \%$ & $52,1 \%$ & $61,7 \%$ & $58,1 \%$ & $60,7 \%$ & $56 \%$ \\
\hline
\end{tabular}

Note de lecture : $60 \%$ des mères isolées propriétaires qui n'ont pas changé de logement au moment du changement familial sont satisfaites de leur logement contre $27,4 \%$ des mères propriétaires qui ont déménagé dans le parc locatif privé.

* La distance moyenne entre logement et lieu de travail pour l'ensemble des mères isolées est d'environ $12 \mathrm{~km}$

** Champ: mères isolées utilisant un service de garde pour leur(s) enfant(s). 


\section{Les déplacements sous contrainte pour le travail et les enfants}

C'est à partir du logement que se structurent les déplacements quotidiens des mères isolées. Près de la moitié d'entre elles estiment que leurs trajets quotidiens se sont complexifiés après leur entrée en monoparentalité, la principale raison invoquée étant la nécessité d'assumer «du jour au lendemain » l'ensemble des déplacements. Les difficultés sont fortes quand la voiture familiale appartenait à l'ex-conjoint ou que les enfants sont jeunes. Les déplacements incontournables parce qu'inhérents aux besoins du ménage - travail de la mère et prise en charge des enfants -, ne font pas exception.

Les déplacements domicile-travail sont un élément majeur dans la dimension économique de l'espace de vulnérabilité des mères seules, qui est celle où la dimension du genre joue le plus fortement. Le fait d'être une mère seule ne vientil pas limiter les capacités à se déplacer pour rejoindre les lieux d'emploi effectifs ou potentiels, et, de ce fait, renforcer les écarts avec l'ensemble des femmes pour lesquelles il a été montré que leur insertion et leur progression dans le marché du travail ont été associées à l'amélioration de l'accessibilité des emplois par automobile à partir des zones de résidence (Vandersmissen et al., 2001, p. 301) ? Les frais de transport et le choix du lieu de travail sont des enjeux majeurs tels que la faiblesse des moyens financiers peut obliger à restreindre l'amplitude et le nombre des déplacements quotidiens. Dans la mesure où elles peuvent difficilement choisir la localisation du domicile en fonction de celle du lieu de travail, les mères isolées les plus fragilisées peuvent être conduites à chercher un emploi proche de leur domicile au détriment des possibilités d'épanouissement ou de promotion. Une mère sur cinq a changé d'emploi au moment de l'entrée en monoparentalité, mais cette valeur moyenne cache des écarts importants entre les mères qui résident dans le parc social et celles qui étaient propriétaires et le sont restées. En conséquence de la recherche de proximité entre domicile et travail, la distance au lieu de travail des mères qui ont dû changer de logement est plus courte que celle des mères n'ayant jamais changé de logement, et notamment des propriétaires (fig. 7). En contrepartie, dans la mesure où elles n'ont changé ni d'emploi ni de domicile et qu'elles ont préservé leur statut résidentiel, la vie de ces dernières a été moins bousculée que celle des femmes qui ont changé d'emploi et connu une régression de leur statut résidentiel. Les mobilités quotidiennes sont alors mieux acceptées. Ces données se combinent avec l'âge et la situation matrimoniale antérieure: les mères n'ayant jamais vécu en couple et qui ont de jeunes enfants habitent davantage à proximité de leur lieu de travail que les mères divorcées, séparées ou veuves ou que, par ailleurs, les mères d'enfants adolescents.

Les déplacements pour le ou les enfants sont d'abord des déplacements vers l'école ou les services de garde. La proximité des écoles et des structures de 
garde est d'ailleurs une souvent invoquée par les mères isolées pour expliquer le choix de leur logement actuel. Les modes de garde varient selon l'offre locale et les ressources que les mères peuvent trouver auprès des ex-conjoints, de leur famille ou d'amis. Seulement un quart des mères seules sont satisfaites de l'offre de services de garde de leur commune, les difficultés mises en avant étant, comme pour toutes les familles, le coût trop élevé, l'inadaptation des horaires ou l'absence d'offre pour des besoins de gardes ponctuelles. Là encore, les propriétaires qui le sont restées sont plus souvent que les autres mères satisfaites de la solution de garde trouvée à l'inverse des femmes qui résident dans le parc HLM. Cela ne permet pas d'affirmer que l'offre à laquelle ces dernières ont recours soit de moins bonne qualité mais qu'elle ne correspond pas à leurs attentes, dont on sait qu'elles sont socialement différenciées entre priorité à l'épanouissement et la socialisation de l'enfant ou à sa prise en charge matérielle (Croutte, Hatchuel, 2003).

La question de la conciliation entre vie professionnelle et vie familiale se pose avec une acuité particulière pour les mères seules (David, 2007). L'emploi d'un temps précieux incite à réduire la longueur des déplacements quotidiens et restreint l'accessibilité aux services. Les contraintes d'emploi du temps quotidien ont donc des conséquences sur les mobilités résidentielles autant qu'elles découlent de ces localisations résidentielles, celles-ci pouvant être guidées par le souci de la proximité avec le domicile de l'ancien conjoint. Pour les mères divorcées ou séparées, la localisation de l'ancien conjoint a un rôle polarisant lorsque la garde de l'enfant est alternée entre les deux parents. D'ailleurs les mères ayant la garde alternée de leur(s) enfant(s) résident plus près de leur ex-conjoint que les mères en ayant la responsabilité à plein-temps. La double résidence du ou des enfant(s) peut cependant être ressentie comme une contrainte en termes de déplacements: les mères partageant la garde de leur(s) enfant(s) sont plus nombreuses que les mères ayant la garde à temps plein à estimer que leurs trajets quotidiens se sont complexifiés. La double résidence des enfants induit des déplacements dont le nombre est fonction de la fréquence et des rythmes des contacts que les enfants ont avec leur père (le plus souvent une semaine sur deux mais aussi selon des jours spécifiques dans la semaine, selon sa disponibilité, etc.).

\section{La voiture, les amis, la famille comme ressources pour se déplacer}

Les mobilités quotidiennes dépendent d'un système d'opportunités, d'aspirations spécifiques et de ressources personnelles (revenus, réseaux sociaux, moyens de locomotion). Les taux de motorisation, les temps de trajet et la propension à solliciter leur entourage proche pour leurs besoins et ceux de leur(s) enfant(s), ou la possibilité de le faire, différencient les mères seules des autres mères mais aussi les mères seules entre elles. 
L'utilisation d'une voiture personnelle est indispensable, surtout lorsque les enfants ne sont pas autonomes dans leur déplacement ou que les mères résident dans une commune rurale. Le fait que les mères isolées soient seules à organiser l'ensemble des déplacements quotidiens fait la part de celles qui disposent d'une voiture personnelle est importante (81,6 \% d'entre elles), même si elle est légèrement inférieure à la moyenne de l'ensemble des ménages de la région (en 2004, $85 \%$ d'entre eux possédaient une voiture ${ }^{10}$. Mais, dans le même temps, la dégradation de la situation financière constatée avec le changement familial (les deux-tiers des mères isolées en témoignent) entraîne des difficultés à se déplacer. La majorité des mères qui ne disposent pas de voiture personnelle le justifient par des raisons financières, qu'il s'agisse du coût de la voiture elle-même ou de celui de son entretien.

Le taux d'équipement automobile diffère selon le niveau de vie (Leray, 2010) mais aussi selon le statut d'occupation. Alors que les propriétaires ou anciennement propriétaires utilisent quasiment toutes une voiture pour se rendre au travail, c'est moins le cas des mères qui ont toujours été locataires dans le parc public (un quart de non-utilisatrices). L'absence de voiture favorise l'isolement physique et social. Obstacle pour accéder à l'emploi, elle peut entraver la participation des mères et des enfants à des activités en dehors du domicile, voire entretenir des processus d'exclusion (Le Breton, 2005). Les mobilités résidentielles vers les pôles urbains, dont nous avons vu qu'elles pouvaient tenir à la présence du parc social, permettent une plus grande accessibilité aux réseaux de transports en commun, au moins pour les principaux pôles urbains que sont Brest et Rennes. Malgré tout, les transports en commun n'offrent ni la continuité de disponibilité ni la commodité de la voiture, tout particulièrement quand il faut se déplacer avec de jeunes enfants.

Quant aux temps de déplacement quotidiens estimés par les mères enquêtées, ils sont très variables. Près de $30 \%$ de celles qui ont la garde totale de leur(s) enfant(s) évaluent leur temps de déplacement à 15 à 30 minutes par jour mais elles sont plus nombreuses à estimer que ce temps dépasse 45 minutes. Les déplacements quotidiens de plus de 45 minutes concernent davantage les mères ayant la garde alternée de leur(s) enfant(s) que les mères en ayant la garde totale. Les déplacements de plus de 45 minutes par jour concernent également davantage les mères ayant de jeunes enfants $(41,5 \%$ des mères ayant des enfants de moins de 11 ans, âge de fin de scolarité primaire) que les mères ayant des enfants plus âgés et donc plus autonomes dans leurs déplacements ( $35 \%$ mères ayant des enfants de 11 ans et plus). Les déplacements de plus d'une heure par jour concernent plus souvent des mères vivant dans une commune rurale (près du quart d'entre elles contre $17 \%$ des mères résidant dans une ville-centre).

10. Insee, recensement de population, 2006. 
Confrontées à des difficultés pour se déplacer, les mères seules peuvent être amenées à faire, régulièrement ou non, appel à leur entourage pour les déplacements de leur(s) enfant(s). Dans l'ensemble, ce sont les mères qui n'ont jamais vécu en couple et dont les enfants sont jeunes qui mobilisent le plus ce type de solidarité à l'inverse des veuves qui sont plus systématiquement motorisées et dont les enfants sont plus âgés. Les sollicitations se retrouvent, à des niveaux plus faibles, pour les déplacements personnels: alors que près du tiers des mères n'ayant jamais vécu en couple sollicitent leur entourage proche, cela n'est le cas que d'une veuve sur cinq. Les différences selon les parcours et statuts résidentiels sont marquées. Les femmes propriétaires de leur logement qui n'ont jamais déménagé sollicitent peu leur entourage pour les déplacements personnels mais, à l'inverse, elles mobilisent davantage que les autres leurs réseaux sociaux pour les déplacements de leur(s) enfant(s). Cela tient sans doute à une combinaison entre plus grande fréquence des activités extrascolaires des enfants, éloignement entre domicile et lieux d'activités, solidarités de voisinage. Par contre, celles qui étaient propriétaires et qui ont emménagé dans un logement HLM font rarement appel à leur entourage pour les déplacements de leurs enfants, parce qu'elles sont plus motorisées que les autres résidantes du parc HLM mais sans doute aussi par manque de confiance à l'égard d'un cadre de vie et d'un voisinage qu'elles subissent plus qu'elles ne l'ont choisi.

\section{La restriction des réseaux sociaux et la rétraction des espaces de vie}

Les mères de familles monoparentales organisent leurs espaces de vie quotidiens selon des arbitrages entre deux priorités:

- préserver la sociabilité de l'enfant par la proximité entre le lieu de résidence, la famille et/ou l'ex-conjoint et ses lieux d'activités (école et/ou loisirs). Quels que soient les arrangements, l'objectif est alors de ne pas bouleverser le cade de vie habituel de l'enfant: "J'ai organisé ma vie en fonction de mon fils et en privilégiant une qualité de vie dans un cadre restreint en termes de kilomètres afin de limiter les dépenses. Mes parents sont un peu loin à mon goût (30 km) » (Anne, séparée, 48 ans, un enfant);

- assurer à la famille des conditions de vie aussi satisfaisantes que possible, quitte à choisir un emploi moins apprécié mais plus proche du domicile. Près d'une mère isolée sur deux considère que sa situation familiale est un handicap pour sa vie professionnelle. Cela concerne davantage les mères qui résident dans un logement HLM, et tout particulièrement celles qui y sont venues avec le changement de situation familiale.

Ainsi, l'exigence de proximité laisse à penser que la monoparentalité entraîne une rétraction des espaces de vie comme l'a exprimé Sophie (divorcée, 39 ans, un enfant): 
«Après le divorce, il a fallu que je réorganise toute ma vie, en pensant d'une autre manière. L'important était que mon enfant soit le moins perturbé possible. C'est pour cela qu'il n'a pas changé d'école alors que moi et son père avons déménagé. On s'était mis d'accord pour ne pas vivre éloignés, mais c'est lui qui a décidé de là où il voulait vivre, pas moi. Il a fait ce qu'il voulait. Donc, j'ai dû trouver un logement pas cher, proche de son logement, mais aussi de l'école. Comme je travaillais avec mon ex-conjoint, j'ai dû aussi changer de travail et trouver un boulot pas très intéressant. Mais bon, au moins, il est à côté de chez nous et permet de vivre dignement même si ce n'est pas facile tous les jours [...]. J'ai déménagé à nouveau trois ans après la séparation parce que le quartier ne me plaisait pas. Je vis désormais dans un autre quartier plus tranquille mais que de difficultés à devoir, encore, trouver un logement peu coûteux et pas trop éloigné de l'école et du père de mon enfant! »

Cette rétraction spatiale s'accompagne fréquemment d'une restriction des réseaux sociaux. Si les épisodes de monoparentalité débouchent dans plus des deux tiers des cas, et sans distinction selon les statuts résidentiels, sur un resserrement des liens avec la famille, ils ont aussi pour effet de mettre à rude épreuve la sociabilité des mères seules. Le changement familial remet en question les relations avec le réseau d'amis. Dans l'ensemble, près de six femmes sur dix estiment que leur nombre d'amis a baissé et, pour une mère sur deux, les amis d'aujourd'hui ne sont pas ceux d'avant. Ne font exception que celles qui étaient propriétaires et le sont restées. Le manque de temps induit une réduction des activités extrafamiliales pour une grande majorité de mères seules. Les arrangements, qui peuvent passer par l'emploi à temps partiel et l'entraide de l'entourage, ne suffisent pas à empêcher la restriction des activités de loisirs à l'extérieur du domicile. Les contraintes d'emploi du temps, surtout pour celles qui travaillent à temps plein, font que près du quart des mères considèrent ne pas accorder assez de temps à leur(s) enfant(s) et que plus de la moitié estiment que leur temps personnel est très insuffisant.

Cette double rétraction génère d'autant plus un surcroît de vulnérabilité qu'elle est associée à une insatisfaction à l'égard du logement et de son environnement, comme c'est le cas à chaque fois que le logement ne correspond pas aux aspirations, ce qui est fréquent puisque moins de $40 \%$ mères ayant répondu à l'enquête sont satisfaites de leur logement, les motifs d'insatisfaction les plus cités étant l'inconfort, l'insalubrité et le prix, et que moins de la moitié sont satisfaites de leur cadre de vie. Ici encore, les écarts sont particulièrement marqués entre celles qui étaient propriétaires et le sont restées et les autres. Sans surprise, les plus critiques à l'égard de logement et de leur cadre de vie sont celles qui sont passées du statut de propriétaires à celui de locataire dans le parc HLM, dont elles deviennent captives. Le sentiment de « captivité » qu'elles expriment révèle 
des territorialités du repli qui combinent blocage des migrations résidentielles, affaiblissement de la mobilité et rétrécissement de l'espace des pratiques quotidiennes (Sélimanowski, 2008, p. 239).

\section{CONCLUSION}

La forte augmentation de la part des familles monoparentales dans le total des familles avec enfants exprime à la fois la fragilité croissante des couples et la non-linéarité des statuts matrimoniaux, d'autant que des recompositions familiales mettent souvent un terme aux épisodes de monoparentalité. Le changement de regard porté sur des situations qui se banalisent n'empêche pas la vulnérabilité des familles monoparentales. Comme le montre la recherche, les mobilités sont une composante de cette vulnérabilité. Les parcours résidentiels régressifs, avec de fréquents passages du statut de propriétaire à celui de locataire, notamment HLM, et de la maison individuelle à l'appartement ou encore la stabilité dans le parc social, sont plus fréquents que les situations de préservation du statut de propriétaire. Les mères seules avec enfants sont souvent obligées de déménager et leurs choix résidentiels sont contraints par le coût du logement et par la recherche de proximité avec le lieu de travail ou le domicile de l'ex-conjoint. C'est que les enfants sont largement au cœur des arbitrages en matière de localisations résidentielles, comme ils le sont plus encore pour l'organisation des temps, des rythmes et des déplacements quotidiens. Pour la vie au quotidien, les contrastes sont importants entre celles qui ont pu rester propriétaires de leur logement et les autres. Les premières ont moins souvent dû changer d'emploi, elles sont plus souvent satisfaites des services de garde pour leurs enfants, elles possèdent plus systématiquement une voiture et, de ce fait, disposent de plus d'autonomie de déplacement et dépendent moins souvent des transports en commun ou d'aides ponctuelles. Dans le même temps, elles sont celles qui mobilisent le plus les solidarités de voisinage pour leurs déplacements et celles qui ont le moins souvent connu une restriction de leurs réseaux sociaux. Les deux seuls éléments apparemment négatifs pour les propriétaires qui le sont restées, à savoir la plus grande longueur des distances à parcourir chaque jour et la plus longue durée des déplacements quotidiens, peuvent être interprétés positivement. Ils témoignent d'espaces de vie plus larges comparativement aux territorialités restreintes de celles qui ont rejoint un logement HLM et dont les sociabilités ont été affectées par le passage à la monoparentalité.

Finalement, nous distinguons quatre types de mobilité résidentielle qui ne sont pas sans conséquence sur la spatialisation des modes de vie. Cela confirme que les individus s'inscrivent dans un système de mobilité et que les mères seules recherchent une certaine cohérence dans leurs désirs de mobilité. Ces quatre types de comportements résidentiels sont les suivants: des mobilités résidentielles 
de longue distance (changement de quartier ou de communes) pour se rapprocher d'un environnement familial ou familier. En conséquence, les pratiques sont confinées dans un espace restreint pour les femmes qui s'appuient sur une sociabilité locale et dans un espace élargi pour celles qui privilégient la qualité de vie (urbaine ou rurale), quitte à devoir être plus mobiles; des mobilités résidentielles de longue distance pour changer de statut d'occupation, notamment accéder au logement social dans une ville-centre. En conséquence, peu de déplacements, peu de sorties et de loisirs sont possibles pour les mères à la mobilité résidentielle descendante; des mobilités résidentielles de proximité qui témoigne d'un ancrage résidentiel en situation de précarité (stabilité dans un parc social). L'ancrage résidentiel bien présent pour les mères les plus vulnérables se poursuit donc en situation monoparentale; des mobilités résidentielles de proximité qui témoigne d'une dépendance à l'environnement social et donc d'un attachement au local. Les pratiques sont concentrées autour du domicile. Nous considérons que l'immobilité résidentielle est subie lorsque les ressources financières limitent sa localisation ou l'accession à un statut d'occupation plutôt qu'un autre. L'immobilité résidentielle serait davantage choisie lorsque le souhait de "rester à proximité » témoigne de la volonté pour les familles de conserver le même cadre de vie et le même tissu relationnel.

Les mobilités résidentielles régressives de la majorité des mères seules sont donc à interpréter comme des indicateurs de réduction des capacités. Il ne faut certes pas généraliser, notamment parce que la fréquence et la qualité des relations avec le père des enfants et l'âge de ceux-ci introduisent des différenciations et que les restrictions spatiales qui imposent un immobilisme contraint peuvent s'estomper lorsque l'enfant gagne en autonomie de déplacement. Cependant, l'étude des mobilités résidentielles et quotidiennes des mères seules a confirmé l'intérêt heuristique du modèle de l'espace de vulnérabilité des familles monoparentales. Même si la monoparentalité n'est qu'une séquence de vie, il est incontestable qu'elle peut, d'une part, produire des ruptures dans les parcours résidentiels et professionnels, et, d'autre part, réduire les sociabilités et les espaces de vie. Les femmes seules avec enfant(s) sont alors confrontées à des dynamiques spatiales qui favorisent la pauvreté économique, la marginalisation, l'exclusion sociale, en cohérence avec le modèle d'espace de vulnérabilité. 